\title{
On m-Full Powers of Parameter Ideals
}

\author{
Naoyuki MATSUOKA \\ Meiji University \\ (Communicated by R. Tsushima)
}

\begin{abstract}
Let $Q$ be a parameter ideal in a Noetherian local ring $A$ with the maximal ideal $\mathfrak{m}$. Then $A$ is a regular local ring and $\mathfrak{m} / Q$ is cyclic, if depth $\mathrm{A}>0$ and $Q^{n}$ is $\mathfrak{m}$-full for some integer $n \geq 1$. Consequently, $A$ is a regular local ring and all the powers of $Q$ are integrally closed in $A$ once $Q^{n}$ is integrally closed for some $n \geq 1$.
\end{abstract}

\section{Introduction}

Let $A$ be a Noetherian local ring with the maximal ideal $\mathfrak{m}$ and $d=\operatorname{dim} A$. Let $I$ be an ideal in $A$. Then we say that $I$ is $\mathfrak{m}$-full if $\mathfrak{m} I: x=I$ for some $x \in \mathfrak{m}$. The notion of $\mathfrak{m}$-full ideal was introduced by $\mathrm{D}$. Rees and played since integrally closed ideals are $\mathfrak{m}$-full under a certain mild condition ([G2, Theorem (2.4)]), an important role in the analysis of integrally closed ideals (cf. [G2, GH1, GH2, GHK, HUV, MTV]).

The present purpose is to prove the following.

THEOREM 1.1. Let $A$ be a Noetherian local ring with the maximal ideal $\mathfrak{m}$ and let $Q$ be a parameter ideal in A. Assume that depth $A>0$ and $Q^{n}$ is $\mathfrak{m}$-full for some integer $n \geq 1$. Then the local ring $A$ is regular and $\mathfrak{m} / Q$ is cyclic.

This theorem provides a new sight of $\mathfrak{m}$-full powers of parameter ideals and gives rise to a sufficiently simple proof of the following result, which has been known if $A$ is excellent and $n$ is sufficiently large ([MTV, Théorème 3]) or if depth $A>0$ [HUV, Corollary 2.11]).

COROLlARY 1.2. Let A be a Noetherian local ring with the maximal ideal $\mathfrak{m}$ and let $Q$ be a parameter ideal in A. Then the following three conditions are equivalent to each other.

(1) A is a regular local ring and $\mathfrak{m} / Q$ is cyclic.

(2) $Q$ is integrally closed in A.

(3) $Q^{n}$ is integrally closed in A for some $n \geq 1$.

When this is the case, the ideals $Q^{\ell}$ are integrally closed in A for all integers $\ell \geq 1$.

Received January 11, 2005

1991 Mathematics Subject Classification. Primary 13H05, Secondary 13H15.

Key words and phrases: generalized Cohen-Macaulay ring, Cohen-Macaulay ring, Gorenstein ring, regular local ring, local cohomology, m-full ideal, multiplicity. 
In Corollary (1.2) our contribution is the implication $(3) \Rightarrow(2)$; the equivalence of conditions (1) and (2) is due to [G2, Theorem (3.1)] as well as the last assertion. Thus, as for the parameter ideals $Q$ in a Noetherian local ring $A$, the integral closedness of any power of $Q$ implies that of all the powers of $Q$ and the regularity of $A$ as well.

A global version of Corollary (1.2) is as follows. We suspect that the assumption $\operatorname{Ass}_{A} A / I=\operatorname{Min}_{A} A / I$ in condition (2) of Proposition (1.3) is superfluous.

Proposition 1.3. Let $A$ be a Noetherian ring. Let $I$ be an ideal in $A$ and assume that $\mu_{A}(I)=h t_{A} I$, where $\mu_{A}(I)$ and $h t_{A} I$ denote the number of generators and the height of $I$, respectively. Then the following conditions are equivalent.

(1) I is integrally closed in A.

(2) $\operatorname{Ass}_{A} A / I=\operatorname{Min}_{A} A / I$ and $I^{n}$ is integrally closed in $A$ for some integer $n \geq 1$. When this is the case, $I^{\ell}$ is integrally closed in A for every integer $\ell \geq 1$.

The proof of Theorem (1.1) and Corollary (1.2) shall be given in Section 3. Section 2 is devoted to some preliminaries. In our proof of Theorem (1.1), some results on Ratliff-Rush closures, FLC rings (that is, generalized Cohen-Macaulay local rings), and $\mathfrak{m}$-full ideals will play key roles, which we will briefly summarize in Section 2.

In what follows, otherwise specified, let $A$ be a Noetherian local ring with the maximal ideal $\mathfrak{m}$ and $d=\operatorname{dim} A$. Let $\mu_{A}(*)$ and $\ell_{A}(*)$ denote the number of generators and the length, respectively. For each ideal $I$ in $A$ let ht $_{A}(I)$ be the height of $I$. We denote by e $(A)=\mathrm{e}_{\mathfrak{m}}^{0}(A)$ the multiplicity of $A$ with respect to the maximal ideal $\mathfrak{m}$. Let $\mathrm{H}_{\mathfrak{m}}^{i}(*)(i \in \mathbf{Z})$ stand for the $i \underline{t h}$ local cohomology functor of $A$ with respect to $\mathfrak{m}$.

\section{Preliminaries}

Let $A$ be a commutative Noetherian ring and let $\mathcal{F}_{A}$ denote the set of ideals in $A$ which contain at least one nonzerodivisor in $A$. For each $I \in \mathcal{F}_{A}$ let

$$
\tilde{I}=\bigcup_{n \geq 0}\left(I^{n+1} I^{n}\right)
$$

be the Ratliff-Rush closure of $I$. Then $I \subseteq \tilde{I} \subseteq \bar{I}$ and $\tilde{I}=\tilde{\tilde{I}}$ (cf. [Mc, Lemma 8.2 (vi)]), where $\bar{I}$ denotes the integral closure of $I$.

Proposition 2.1. (1) (Y. Shimoda) Let $I \subseteq J$ be ideals in $A$ and assume that $I^{n}=J^{n}$ for some integer $n \geq 1$. Then $I^{\ell}=J^{\ell}$ for all integers $\ell \geq n$.

(2) Let $I \in \mathcal{F}_{A}$ and assume that $I^{n}=\bar{I}^{n}$ for some $n \geq 1$. Then $\bar{I}=\tilde{I}$ and $I^{\ell}=\bar{I}^{\ell}$ for all integers $\ell \geq n$.

Proof. (1) Since $I^{n} \subseteq I^{n-1} J \subseteq J^{n-1} J=J^{n}$, we get $I^{n}=I^{n-1} J=J^{n}$. Therefore $I^{n+1}=I I^{n}=I\left(I^{n-1} J\right)=I^{n} J=J^{n} J=J^{n+1}$. Thus $I^{\ell}=J^{\ell}$ for all $\ell \geq n$.

(2) We have $I^{n}=\bar{I}^{n}$ since $\bar{I}^{n} \subseteq \overline{I^{n}}=I^{n}$. Hence $\bar{I} \subseteq \tilde{I}$ by [Mc, Lemma 8.2 (iv)] so that $\bar{I}=\bar{I}$ by [Mc, Lemma 8.2 (vi)]. The latter equality follows from assertion (1). 
Now let $A$ be a Noetherian local ring with the maximal ideal $\mathfrak{m}$ and $d=\operatorname{dim} A$. Let $\mathrm{H}_{\mathfrak{m}}^{i}(*)(i \in \mathbf{Z})$ be the local cohomology functors of $A$ with respect to $\mathfrak{m}$. Then we say that $A$ has FLC (or equivalently, $A$ is a generalized Cohen-Macaulay local ring), if all the local cohomology modules $\mathrm{H}_{\mathfrak{m}}^{i}(A)(i \neq d)$ are finitely generated.

For each ideal $I$ in $A$ we put

$$
\mathcal{R}(I)=A[I t]=\bigoplus_{n \geq 0} I^{n},
$$

where $t$ denotes an indeterminate. Let

$$
\mathrm{G}(I)=\mathcal{R}(I) / I \mathcal{R}(I)=\bigoplus_{n \geq 0} I^{n} / I^{n+1}
$$

be the associated graded ring of $I$.

Let $G=\mathrm{G}(\mathfrak{m})$ and $M=G_{+}$the unique graded maximal ideal in $G$. Let $\mathrm{e}(A)=\mathrm{e}_{\mathfrak{m}}^{0}(A)$ denote the multiplicity of $A$. We then have the following.

PROPOSITION 2.2. (1) Suppose that $A$ has FLC. Then $A$ is a regular local ring, if $\mathrm{e}(A)=1$ and $\operatorname{depth} A>0$.

(2) The local ring $A$ has FLC, if the local cohomology modules

$$
H_{M}^{i}(G)=\lim _{n \rightarrow \infty} \operatorname{Ext}_{G}^{i}\left(G / M^{n}, G\right)
$$

of $G$ with respect to $M$ are finitely generated for all $i \neq d$.

Proof. (1) The local ring $A$ is unmixed since $A$ has FLC and depth $A>0$ (cf. [SV, Appendix, Proposition 16]). Hence $A$ is regular by [N, Theorem 40.6].

(2) See [G1, Proposition (3.1)]).

The notion of $\mathfrak{m}$-full ideal was introduced by D. Rees, who showed that every integrally closed ideal $I$ is $\mathfrak{m}$-full, provided $I$ is not nilpotent and the residue class field $A / \mathfrak{m}$ of $A$ is infinite [G2, Theorem (2.4)]). The readers may consult [G2] about basic results on $\mathfrak{m}$-full ideals. Here let us note two of them, which we later need to prove Theorem (1.1).

Proposition 2.3. Let I be an ideal in A and assume that $I$ is $\mathfrak{m}$-full. Then the following assertions hold true.

(1) Let $J$ be an ideal in A. Assume $I \subseteq J$ and $\ell_{A}(J / I)<\infty$. Then $\mu_{A}(I) \geq \mu_{A}(J)$.

(2) Assume that $A / I$ is an Artinian Gorenstein local ring. Then $\mathfrak{m} / I$ is cyclic.

Proof. (1) See [G2, Lemma (2.2) (2)].

(2) Let $x \in \mathfrak{m}$ such that $\mathfrak{m} I: x=I$. Then $I: \mathfrak{m}=(\mathfrak{m} I: x): \mathfrak{m}=(\mathfrak{m} I: \mathfrak{m}): x \supseteq I:$ $x$ so that $I: \mathfrak{m}=I: x$. Thus we get the exact sequence

$$
0 \rightarrow[I: \mathfrak{m}] / I \rightarrow A / I \stackrel{\hat{x}}{\rightarrow} \rightarrow A / I \rightarrow A /[I+(x)] \rightarrow 0 .
$$

Hence $\ell_{A}(A /[I+(x)])=\ell_{A}([I: \mathfrak{m}] / I)=1$ because $A / I$ is Gorenstein. Thus $\mathfrak{m}=I+(x)$, whence $\mathfrak{m} / I$ is cyclic. 


\section{Proofs of Theorem 1.1 and Corollary 1.2}

Let $A$ be a Noetherian local ring with the maximal ideal $\mathfrak{m}$ and $d=\operatorname{dim} A$. Let $Q$ be a parameter ideal in $A$.

Proof of Theorem 1.1. Passing to the local ring $A[X]_{\mathfrak{m} A[X]}$ where $X$ is an indeterminate over $A$, we may assume that the residue class field $k=A / \mathfrak{m}$ of $A$ is infinite. Let $\mathfrak{q}$ be a minimal reduction of $\mathfrak{m}$. Hence $\mathfrak{q}$ is a parameter ideal in $A$ and $\mathfrak{m}^{r+1}=\mathfrak{q m}^{r}$ for some $r \geq 0$ (such an ideal $\mathfrak{q}$ must exist because the field $k=A / \mathfrak{m}$ is infinite), and then $\mathcal{R}(\mathfrak{m}$ ) is a module-finite extension of $\mathcal{R}(\mathfrak{q})$. Let

$$
\varphi: \mathcal{R}(\mathfrak{q}) / \mathfrak{m} \mathcal{R}(\mathfrak{q}) \rightarrow \mathcal{R}(\mathfrak{m}) / \mathfrak{m} \mathcal{R}(\mathfrak{m})
$$

be the homomorphism of graded $k$-algebras induced from the inclusion $\mathcal{R}(\mathfrak{q}) \subseteq \mathcal{R}(\mathfrak{m})$. Hence the homomorphism $\varphi$ is also finite. We put

$$
P=\mathcal{R}(\mathfrak{q}) / \mathfrak{m} \mathcal{R}(\mathfrak{q}) \quad \text { and } \quad G=\mathcal{R}(\mathfrak{m}) / \mathfrak{m} \mathcal{R}(\mathfrak{m}) .
$$

For each integer $i \geq 0$ let $P_{i}=\mathfrak{q}^{i} / \mathfrak{m} \mathfrak{q}^{i}$ and $G_{i}=\mathfrak{m}^{i} / \mathfrak{m}^{i+1}$ denote the homogeneous components of $P$ and $G$ of degree $i$. Then because $\mathfrak{q}$ is a parameter ideal in $A$, the ring $P$ is the polynomial ring with $d$ variables over the field $k$. Therefore $\varphi$ is a monomorphism since $\varphi$ is finite and $\operatorname{dim} P=\operatorname{dim} G=d$.

We now look at the $\mathfrak{m}$-full ideal $Q^{n}$. Then

$$
\mu_{A}\left(\mathfrak{m}^{n}\right) \leq \mu_{A}\left(Q^{n}\right)=\left(\begin{array}{c}
d+n-1 \\
d-1
\end{array}\right)=\mu_{A}\left(\mathfrak{q}^{n}\right)
$$

by Proposition (2.3) (1), so that the monomorphism $\varphi$ induces an isomorphism

$$
P_{n}=\mathfrak{q}^{n} / \mathfrak{m} \mathfrak{q}^{n} \rightarrow G_{n}=\mathfrak{m}^{n} / \mathfrak{m}^{n+1}
$$

of vector spaces over $k$. Hence $\mathfrak{m}^{n}=\mathfrak{q}^{n}+\mathfrak{m}^{n+1}$ and so $\mathfrak{m}^{n}=\mathfrak{q}^{n}$ by Nakayama's lemma. Thus $\mathfrak{m}^{\ell}=\mathfrak{q}^{\ell}$ for all integers $\ell \geq n$ by Proposition (2.1) (1). Hence the homomorphism $\varphi: P \rightarrow G$ induces an isomorphism between the vector spaces $P_{\ell}$ and $G_{\ell}$ over $k$ and so

$$
\ell_{A}\left(\mathfrak{m}^{\ell} / \mathfrak{m}^{\ell+1}\right)=\ell_{A}\left(\mathfrak{q}^{\ell} / \mathfrak{m} \mathfrak{q}^{\ell}\right)=\left(\begin{array}{c}
d+\ell-1 \\
d-1
\end{array}\right)
$$

for every $\ell \geq n$. Thus $\mathrm{e}(A)=\mathrm{e}_{\mathfrak{m}}^{0}(A)=1$ by definition. Let $C=\operatorname{Coker} \varphi$. Then $\operatorname{dim}_{k} C<\infty$ since $C_{\ell}=(0)$ if $\ell \geq n$. Therefore because the ring $P$ is the polynomial ring over $k$, thanks to the exact sequence

$$
0 \rightarrow P \stackrel{\varphi}{\rightarrow} \rightarrow G \rightarrow C \rightarrow 0
$$

of finitely generated graded $P$-modules, we get $\mathrm{H}_{M}^{i}(G)=(0)$ for all $i \neq 0, d$, where $M=$ $G_{+}$. Hence by Proposition (2.2) (2) the local ring $A$ has FLC, so that the local ring $A$ is regular by Proposition (2.2) (1) because e $(A)=1$ and depth $A>0$. 
Since $A / Q$ is an Artinian Gorenstein local ring, to see that $\mathfrak{m} / Q$ is cyclic, by Proposition (2.3) (2) it is enough to show that $Q$ is $\mathfrak{m}$-full. Let $x \in \mathfrak{m}$ such that $\mathfrak{m} Q^{n}: x=Q^{n}$ and let $a \in \mathfrak{m} Q: x$. Then $x\left(a Q^{n-1}\right)=(x a) Q^{n-1} \subseteq \mathfrak{m} Q^{n}$ whence $a Q^{n-1} \subseteq \mathfrak{m} Q^{n}: x=Q^{n}$. Therefore $a \in Q^{n}: Q^{n-1}=Q$ because $Q$ is generated by an $A$-regular sequence. Thus $\mathfrak{m} Q: x=Q$ and so $Q$ is $\mathfrak{m}$-full.

We are in a position to prove Corollary (1.2). The last assertion and the equivalence of conditions (1) and (2) in Corollary (1.2) are due to [G2, Theorem (3.1)]. Let us include brief proofs of the last assertion and the implication $(2) \Rightarrow(1)$ for the sake of completeness.

Proof of Corollary 1.2. We may assume that $d=\operatorname{dim} A>0$. Passing to the local ring $A[X]_{\mathfrak{m} A[X]}$ where $X$ is an indeterminate over $A$, we may also assume that the residue class field $k=A / \mathfrak{m}$ of $A$ is infinite.

(3) $\Rightarrow$ (2) We will show that $A$ is a regular local ring and $Q$ is integrally closed. Let $W=\mathrm{H}_{\mathfrak{m}}^{0}(A), B=A / W$, and $\mathfrak{n}=\mathfrak{m} / W$. Then $Q^{n} B$ is integrally closed in $B$ because $W \subseteq \sqrt{(0)}$ and $Q^{n}$ is integrally closed in $A$. Hence by Theorem (1.1) the local ring $B$ is regular because depth $B>0$ and $Q^{n} B$ is $\mathfrak{n}$-full. We must show that $W=(0)$. Since $W \subseteq \sqrt{(0)}$, we have $W \subseteq \overline{Q^{n}}=Q^{n}$. Let $\ell>0$ be an integer and assume that $W \subseteq Q^{\ell}$. Let $Q=\left(a_{1}, a_{2}, \ldots, a_{d}\right)$ and choose $w \in W$. We write $w=\sum_{|\alpha|=\ell} c_{\alpha} \mathbf{a}^{\alpha}$ with $c_{\alpha} \in A$, where $\mathbf{a}^{\alpha}=a_{1}^{\alpha_{1}} a_{2}^{\alpha_{2}} \cdots a_{d}^{\alpha_{d}}$ and $|\alpha|=\sum_{i=1}^{d} \alpha_{i}$ for each $\alpha=\left(\alpha_{1}, \alpha_{2}, \ldots, \alpha_{d}\right)$ with $0 \leq \alpha_{i} \in \mathbf{Z}$. Let $\bar{*}$ denote the image in $B$. Then

$$
\sum_{|\alpha|=\ell} \overline{c_{\alpha}}{\overline{a_{1}}}^{\alpha_{1}}{\overline{a_{2}}}^{\alpha_{2}} \cdots{\overline{a_{d}}}^{\alpha_{d}}=\bar{w}=0 .
$$

Since the system $\overline{a_{1}}, \overline{a_{2}}, \ldots, \overline{a_{d}}$ of parameters in $B$ forms a regular sequence, we get $\overline{c_{\alpha}} \in Q B$ for every $\alpha=\left(\alpha_{1}, \alpha_{2}, \ldots, \alpha_{d}\right)$ with $|\alpha|=\ell$. Thus $c_{\alpha} \in Q+W$ so that $w \in\left(Q^{\ell+1}+W Q^{\ell}\right) \cap$ $W=\left(Q^{\ell+1} \cap W\right)+W Q^{\ell}$. Consequently, $W=Q^{\ell+1} \cap W \subseteq Q^{\ell+1}$ by Nakayama's lemma. Hence $W \subseteq \bigcap_{\ell>0} Q^{\ell}=(0)$ and so the local ring $A$ is regular.

Because $Q^{n}$ is integrally closed, we have $\bar{Q}=\tilde{Q}$ by Proposition (2.1) (2). The ideal $Q$ is generated by a regular sequence, whence

$$
\tilde{Q}=\bigcup_{n \geq 0}\left(Q^{n+1}: Q^{n}\right)=Q,
$$

so that we have $\bar{Q}=\tilde{Q}=Q$.

$(2) \Rightarrow(1)$ Thanks to the above proof, $A$ is a regular local ring. Hence $\mathfrak{m} / Q$ is cyclic by Proposition (2.3) (2) because $Q$ is $\mathfrak{m}$-full and $A / Q$ is an Artinian Gorenstein local ring.

$(1) \Rightarrow$ the last assertion. We may assume that $d \geq 2$. Since $\mathfrak{m} / Q$ is cyclic by our assumption, we may choose a regular system $a_{1}, a_{2}, \ldots, a_{d}$ of parameters of $A$ so that $Q=$ $\left(a_{1}, \ldots, a_{d-1}, a_{d}^{q}\right)$ for some $q \geq 1$. Let

$$
\mathcal{S}=\mathcal{R}(Q)\left[t^{-1}\right]=A\left[a_{1} t, \ldots, a_{d-1} t, a_{d}^{q} t, t^{-1}\right]
$$


be the extended Rees algebra of $Q$ (here $t$ denotes an indeterminate over $A$ ). Let $u=t^{-1}$. Then $\mathrm{G}(Q)=\mathcal{S} / u \mathcal{S}$. We must show that $\mathcal{S}$ is an integrally closed integral domain. We firstly recall that the sequence $a_{1}, \ldots, a_{d-1}, a_{d}^{q}$ is regular. Hence the associated graded ring $\mathrm{G}(Q)$ is the polynomial ring with $d$ indeterminates over $A / Q$, that is

$$
\mathrm{G}(Q)=(A / Q)\left[\overline{a_{1} t}, \ldots, \overline{a_{d-1} t}, \overline{a_{d}^{q} t}\right]
$$

and the elements $\left\{\overline{a_{i} t}\right\}_{1 \leq i \leq d-1}$ and $\overline{a_{d}^{q} t}$ are algebraically independent over $A / Q$, where $\bar{*}$ denotes the image in $\mathrm{G}(Q)$. In particular, the ring $\mathrm{G}(Q)$ is Cohen-Macaulay. Hence the ring $\mathcal{S}$ is also Cohen-Macaulay because $u$ is a nonzero divisor in $\mathcal{S}$.

Let $P$ be a prime ideal in $\mathcal{S}$ with ht ${ }_{\mathcal{S}} P=1$. We will show that the localization $\mathcal{S}_{P}$ of $\mathcal{S}$ is a discrete valuation ring. We may assume that $u \in P$ (because the ring $\mathcal{S}\left[u^{-1}\right]=A\left[t, t^{-1}\right]$ is regular). Then $P=(\mathfrak{m}, u) \mathcal{S}=\left(a_{1}, a_{2}, \ldots, a_{d}, u\right) \mathcal{S}$, since $P / u \mathcal{S}$ is a unique minimal prime ideal in the polynomial ring $\mathrm{G}(Q)$. Hence $P=\left(a_{d}, u\right) \mathcal{S}$ because $a_{i}=a_{i} t \cdot u$ for all $1 \leq i \leq d-1$. Therefore $P \mathcal{S}_{P}=a_{d} \mathcal{S}_{P}$ because $a_{d}^{q} t \notin P=(\mathfrak{m}, u) \mathcal{S}$ and $u=a_{d}^{q} /\left(a_{d}^{q} t\right)$. Hence $\mathcal{S}_{P}$ is a discrete valuation ring with the regular parameter $a_{d}$. Thus the CohenMacaulay ring $\mathcal{S}$ satisfies Serre's condition $\left(\mathrm{R}_{1}\right)$, so that $\mathcal{S}$ is an integrally closed integral domain. Hence $Q^{n}$ is integrally closed in $A$ for every integer $n \geq 1$, which completes the proof of Corollary (1.2).

Before closing this paper let us note a brief proof of Proposition (1.3). We suspect the assumption that $\operatorname{Ass}_{A} A / I=\operatorname{Min}_{A} A / I$ in condition (2) is superfluous.

Proof of Proposition 1.3. (1) $\Rightarrow(2)$ and the last assertion. This is due to [G2, Theorem (1.1)].

(2) $\Rightarrow$ (1) Assume that $I \neq \bar{I}$ and choose $P \in \operatorname{Ass}_{A} \bar{I} / I$. Then $P \in \operatorname{Ass}_{A} A / I=$ $\operatorname{Min}_{A} A / I$. Hence the ideal $I A_{P}$ is a parameter ideal in the local ring $A_{P}$ because $\mathrm{ht}_{A} I=$ $\mu_{A}(I)$. Since

$$
\left(I A_{P}\right)^{n}=I^{n} A_{P}=\overline{I^{n}} A_{P}=\overline{I^{n} A_{P}}=\overline{\left(I A_{P}\right)^{n}},
$$

by Corollary (1.2) the local ring $A_{P}$ is regular and $I A_{P}=\overline{I A_{P}}=\bar{I} A_{P}$. This is impos sible.

\section{References}

[G1] S. Goto, Noetherian local rings with Buchsbaum associated graded rings, J. Alg. 86 (1984), 336-384.

[G2] S. Gото, Integral closedness of complete-intersection ideals, J. Alg. 108 (1987), 151-160.

[GH1] S. Goto and F. HAYASAKA, Finite homological dimension and primes associated to integrally closed ideals, Proc. Amer. Math. Soc. 130 (2002), 3159-3164

[GH2] S. Goto and F. HAYASAKA, Finite homological dimension and primes associated to integrally closed ideals II, J. Math. Kyoto Univ. 42-4 (2002), 631-639. 
[GHK] S. Goto, F. HAYASAKA and S. KASUGA, Towards a theory of Gorenstein m-primary integrally closed ideals, J. Herzog and V. Vuletescu (eds.): Commutative Algebra, Singularities and Computer Algebra, The Proceedings of NATO Advanced Research Workshop: Sinaia (Romania) (2002), 159-177.

[HUV] S. C. HunEKE, B. UlRiCH and W. V. VASCONCELOS, On the structure of certain normal ideals, Compositio Mathematica 84 (1992) 25-42.

[Mc] S. McAdam, Asymptotic Prime Divisors, Lecture Notes in Math. vol. 1023, Springer-Verlag, Berlin·Heidelberg·New York·Tokyo (1983).

[MTV] M. Morales, N. V. Trung and O. Villamayor, Sur la fonction de Hilbert-Samuel des clôtures intégrales des puissances d'idèaux engendrés par un système de paramètres, J. Alg. 129 (1990), 96-102.

[N] M. NAGATA, Local Rings, Interscience (1962).

[SV] J. StÜCKRAD and W. VogeL, Buchsbaum Rings and Applications, Springer-Verlag (1986).

Present Address:

Department of Mathematics, School of Science and Technology,

MEIJI UNIVERSITY,

HIGASHI-MITA, TAMA-KU, KAWASAKI-SHI, KANAGAWA, 214-8571 JAPAN.

e-mail: matsuoka@math.meiji.ac.jp 